\title{
The Probiotics in Pediatric Asthma Management (PROPAM) Study in the Primary Care Setting: A Randomized, Controlled, Double-Blind Trial with Ligilactobacillus salivarius LS01 (DSM 22775) and Bifidobacterium breve B632 (DSM 24706)
}

\author{
Lorenzo Drago $\left(\mathbb{D},{ }^{1}\right.$ Luigi Cioffi $\left(\mathbb{D},{ }^{2}\right.$ Maria Giuliano, ${ }^{2}$ Marco Pane ${ }^{(D)},{ }^{3}$ Angela Amoruso, ${ }^{3}$ \\ Irene Schiavetti $\mathbb{D}^{4},{ }^{4}$ Gregor Reid $\mathbb{D}^{5},{ }^{5}$ Giorgio Ciprandi $\mathbb{D}^{6},{ }^{6}$ and PROPAM Study Group ${ }^{2}$ \\ ${ }^{1}$ Department of Biomedical Sciences for Health, University of Milan, Milan, Italy \\ ${ }^{2}$ Pediatric Primary Care ASL Napoli 2, Naples, Italy \\ ${ }^{3}$ Research and Development, Probiotical Research, Novara, Italy \\ ${ }^{4}$ Health Science Department, University of Genoa, Genoa, Italy \\ ${ }^{5}$ Departments of Microbiology, Immunology and Surgery, Western University, London, ON, Canada \\ ${ }^{6}$ Allergy Clinic, Casa di Cura Villa Montallegro, Genoa, Italy
}

Correspondence should be addressed to Giorgio Ciprandi; gio.cip@libero.it

Received 1 December 2021; Revised 13 December 2021; Accepted 4 January 2022; Published 17 January 2022

Academic Editor: Baohui Xu

Copyright (c) 2022 Lorenzo Drago et al. This is an open access article distributed under the Creative Commons Attribution License, which permits unrestricted use, distribution, and reproduction in any medium, provided the original work is properly cited.

\begin{abstract}
Background. Type-2 inflammation commonly marks asthma in childhood. Also, gut and lung dysbiosis is detectable in patients with asthma. Strain-related probiotic supplementation may restore a physiological immune response, dampen airway inflammation, and repair dysbiosis. Therefore, the probiotics in pediatric asthma management (PROPAM) study is aimed at demonstrating that Ligilactobacillus salivarius LS01 (DSM 22775) and Bifidobacterium breve B632 (DSM 24706) mixture could reduce asthma exacerbations in children, followed in a primary care setting. Methods. The study was randomized, placebocontrolled, and double-blind. It involved 11 Italian primary care pediatricians. The probiotic mixture (containing Ligilactobacillus salivarius LS01 $1 \times 10^{9}$ live cells and Bifidobacterium breve B632 $1 \times 10^{9}$ live cells) or placebo was taken twice daily ( 1 sachet in the morning and 1 in the evening) for eight weeks and subsequently once daily for a further eight weeks. Outcomes included number, severity, and duration of asthma exacerbations, intensity of maintenance and as need treatments, and safety. Results. The per-protocol population included 422 children (mean age seven years, 240 males and 182 females). The probiotic mixture significantly reduced the number of asthmatic exacerbations $(\mathrm{OR}=3.17)$. In addition, the number of children with two exacerbations was less than a third in the active group $(\mathrm{OR}=3.65)$. Conclusions. This PROPAM study demonstrated that probiotic strains Ligilactobacillus salivarius LS01 (DSM 22775) and Bifidobacterium breve B632 (DSM 24706) were safe and significantly reduced by more than a third the frequency of asthma exacerbations. At present, the firstline treatment of asthma is still drug-based, but specific strains of probiotics may be auxiliary remedies.
\end{abstract}

\section{Introduction}

Asthma, including wheezing, represents a severe global health problem and a relevant burden for the healthcare system, as underscored by the 2021 Global Initiative for
Asthma (GINA) guidelines (www.ginasthma.org). Children with asthma usually have a type- 2 phenotype and consequently are prone to have frequent respiratory infections [1]. Moreover, asthma exacerbations often follow acute respiratory infections in childhood [2]. As a result, 
modulation of the immune response and prevention of respiratory infections assume an essential role in the therapeutical strategy.

The increase in asthma prevalence has been initially attributed to the hygiene hypothesis, especially to the imbalance of human microbiota composition, abundance, and diversity (dysbiosis) that promotes the maintenance of type-2 phenotype in the infant [3]. This gut microbiota dysbiosis appears to play a role in increasing allergy prevalence [4]. It has been proposed that a decline in biodiversity determines microbial deprivation affecting the immune response. Indeed, children with asthma display lung and intestinal dysbiosis [5] likely promote the activation of inflammatory pathways and contribute to bronchial obstruction and airway hyperresponsiveness. Thus, dysbiosis and reduced microbial diversity dysregulate the bidirectional crosstalk across the gut-lung axis [6]. This axis provides a rational for understanding how oral supplements could improve respiratory illness [7]. These concepts have paved the way for manipulating the immune system using nonpharmacological remedies including probiotics.

There is a body of pathophysiological evidence supporting probiotic use in allergy and asthma [8]. Certain strains can promote the expansion of type-1 response, downregulate IgE production, and reinforce the immune defense against respiratory infections [9]. Studies have explored the potential effects of probiotics in preventing allergic diseases and asthma; however, the outcomes were conflicting because of a high degree of heterogeneity among studies, mainly concerning study design, populations, timing, considered variables, and overall used strains [10-13]. It is essential to consider the properties of strains including their genetic, adaptative, immunological, and metabolic characteristics, to better target the desired efficacy and safety. In the present study, the mixture of two well-characterized strains, Bifidobacterium breve B632 and Ligilactobacillus salivarius LS01, was based on microbiological and clinical evidence that supported their potential role in asthma management [14-17]. As a result, the PRObiotics in Pediatric Asthma Management (PROPAM) study tested the hypothesis that these strains could prevent asthma exacerbations in a pediatric primary care setting. The aim was, therefore, to evaluate the possible reduction of asthma exacerbations and improvement of disease severity.

\section{Materials and Methods}

The PROPAM study was designed as a randomized, placebo-controlled, and double-blind trial. The primary outcome was the reduction of asthma exacerbations, considering the number, duration (days), and severity of asthma attacks. The severity was assessed using the scoring provided by the Italian Society of Pediatrics [18]. It was graded as mild, moderate, and severe.

The secondary outcome was the reduction of drugs used in maintenance and as needed for asthma exacerbations.

The variables included (i) the number of asthma exacerbations, severity, and duration documented by parents in a diary and assessed by clinicians at visits; (ii) the maintenance therapy, assessed by the intensity of treatment, scored as follows: no treatment, low intensity (antileukotrienes alone), medium intensity (inhaled corticosteroids alone), high intensity (inhaled corticosteroids combined with longacting $\beta 2$-agonists and/or antileukotrienes), and (iii) the as-needed therapy during exacerbations, considering the use of oral corticosteroid and/or increase of inhaled corticosteroid dosage.

The eligibility criteria consisted of inclusion and exclusion criteria. The inclusion criteria were age between 3 and 14 years, and asthma diagnosis, according to GINA criteria. The exclusion criteria were severe asthma, congenital or acquired immunodeficiency, cystic fibrosis, and chronic pulmonary diseases.

The study started on April 2017 and finished in November 2019. The treatment period lasted 16 weeks.

The study included five visits: at baseline (T0), after one month of treatment (T1), two months (T2), three months (T3), and four months (T4). During each visit, the investigator performed detailed medical history, mainly concerning asthma exacerbations and use of medications, physical examination, drug countability, and revised the therapeutic strategy if necessary.

Each sachet of the active probiotic product comprised viable strains currently used in food supplements, specifically $\geq 1 \times 10^{9}$ live cells of B. breve B632 (DSM 24706) and $\geq 1 \times 10^{9}$ live cells of $L$. salivarius LS01 (DSM 22775) (combined dose of $\geq 2 \times 10^{9}$ live), with maltodextrin used as a bulking agent to yield a final weight of 2 grams; each placebo sachet contained 2 grams of maltodextrin only (Probiotical S.p.A., Novara, Italy). The placebo powder was indistinguishable from the probiotic powder in appearance, taste, smell, and packaging. Participants were instructed to dissolve the powder in water or cold milk and drink it in the morning and evening. The probiotic sachets were analyzed by Biolab Research S.r.l., Novara, Italy, via flow cytometry (ISO 19344:2015 IDF 232:2015, $\geq 2 \times 10^{9}$ active fluorescent units (AFU)) and plate count method (Biolab Research Method 014-06, $\geq 2 \times 10^{9} \mathrm{CFU}$ ) to confirm target cell count.

The sample size was calculated to power the study to detect a $25 \%$ reduction of asthma episodes. This required enrollment of 200 subjects per arm. Assuming a drop-out rate of $20 \%$, a total of 500 children enrolled was judged adequate to provide sufficient quantity to detect the stated percentage. The estimates were obtained, setting the probability of type I error $\alpha=0.05$ (two-tailed) and a CI width of 0.14 . Participants were enrolled and randomized with a 1.1 ratio. The randomization method was computer-generated. Globally, 11 Italian primary care pediatricians participated in the study, each having to enroll 46 children. All of them resided in the Campania (South Italy) region.

The Ethics Committee of the ASL Napoli 3 Sud approved the study procedure on April 12, 2017 (N. 45/21/04/2017).

The statistical assessment included a descriptive analysis of collected data, summarized as counts within a group for categorical variables and with mean \pm standard deviation and median with interquartile range for continuous variables. Univariate logistic regression models were applied to predict the outcomes' likelihood (presence of asthma 


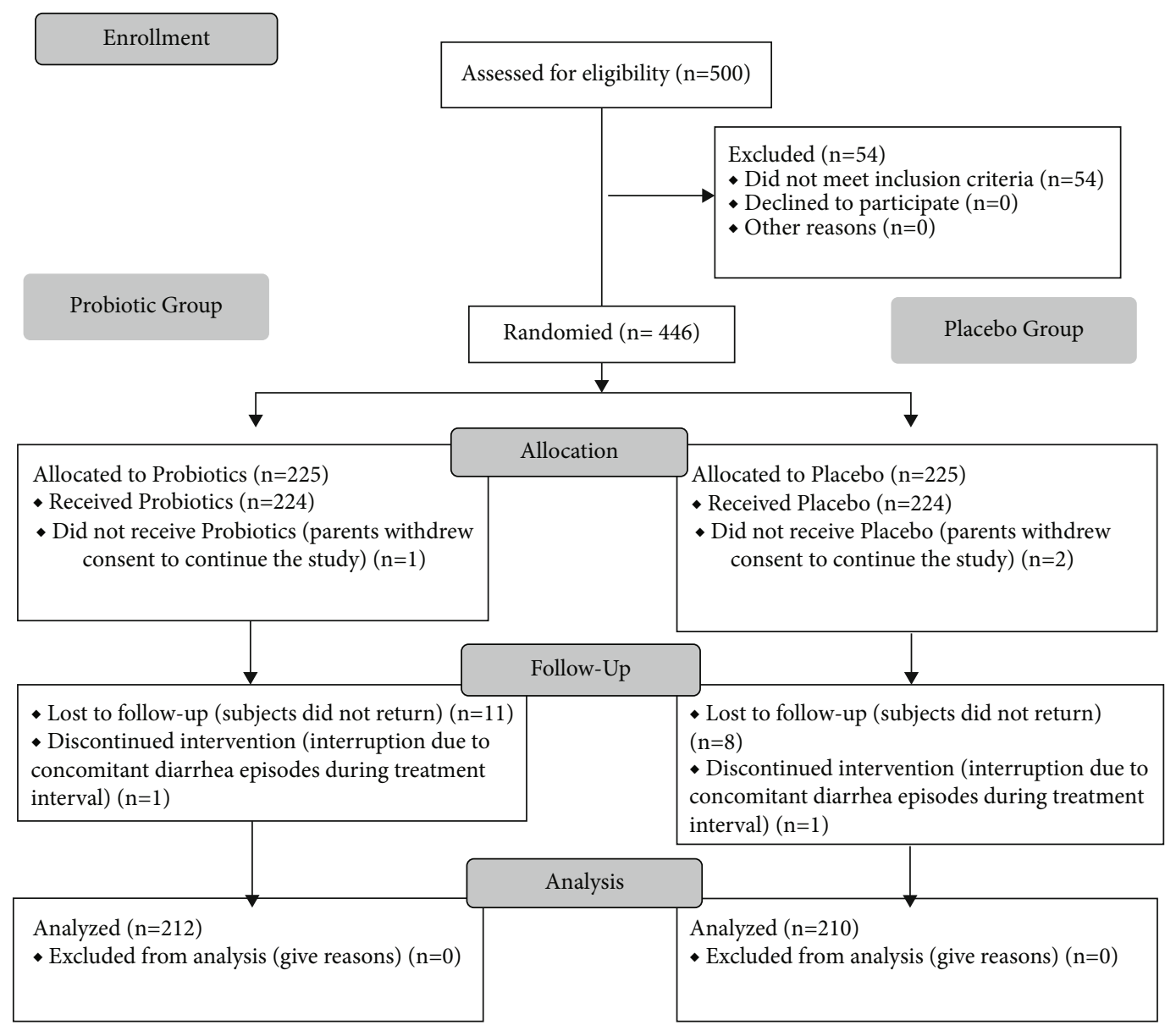

Figure 1: CONSORT flowchart.

exacerbation during the treatment period). Results were quantified by odds ratio (OR) together with a $95 \%$ confidence level $(95 \% \mathrm{CI})$. The significance level was set at 0.05 . The analyses were computed using SPSS Statistics version 21.0 (IBM Corp., Armonk, NY, USA).

\section{Results}

The pediatricians enrolled 500 children who were screened for clinical trial eligibility. Figure 1 reports the patient disposition. The analyzed per-protocol (PP) population consisted of 422 children: 212 in the active arm and 210 in the placebo one.

3.1. General Characteristics. Table 1 summarized the demographic and clinical data in the PP population at baseline (T0). It included 422 children (mean age $7+3.17$ years), 182 females, and 240 males. Of these, 291 resided in the city and 131 in rural communities. One hundred and seventy-six subjects (41.7\%) reported passive smoking. Only 50 (11.8\%) did not go to school. Family atopy was declared for 320 $(75.8 \%)$ children, and sensitization was reported in 210 (49.8\%) children.

The analysis stratified the patient population into an active group and a placebo group. The intergroup comparison showed no significant differences between study groups in all demographic and clinical characteristics at baseline (Table 1). Thus, the two groups were well-matched.

3.2. Primary Outcomes. Fifty (23.8\%) children in the placebo group experienced at least one asthma exacerbation compared to $19(9 \%)$ in the active group (Table 2). Seventeen (8.1\%) children of the placebo group and $5(2.4 \%)$ in the active group had two asthma exacerbations. In total, there were 67 asthma exacerbations in the placebo group and 24 in the active group. The univariate logistic regression analysis showed that children in the placebo group had a higher probability of having at least one asthma exacerbation than children in the probiotic arm: (OR 3.17, 95\% CI 1.8-5.6; $p$ $<0.001)$. In addition, children in the placebo group were more likely to have two exacerbations than children in the active group (OR 3.65, 95\% CI 1.32-10.08; $p=0.013$ ). In other words, the probiotic mixture reduced nearly to a quarter the probability of having two asthma exacerbations.

In terms of severity of asthma exacerbations, children in the placebo group had 21 (31.3\%) mild episodes, 44 (65.7\%) moderate episodes, and $2(3.0 \%)$ severe. In the active arm, 4 (16.7\%) children had mild asthma exacerbations, 19 (79.2\%) moderate, and 1 (4.1\%) severe (Table 3). The mean asthma exacerbation duration was $3.3+2.57$ days in the placebo group and $3.3+2.45$ in the active arm (Table 3 ). 
TABLE 1: Baseline characteristics.

\begin{tabular}{|c|c|c|c|}
\hline & $\begin{array}{c}\text { Total } \\
N=422\end{array}$ & $\begin{array}{l}\text { Placebo } \\
N=210\end{array}$ & $\begin{array}{c}\text { Active treatment } \\
\qquad N=212\end{array}$ \\
\hline Age & $7.0 \pm 3.17$ & $7.0 \pm 2.95$ & $7.0 \pm 3.38$ \\
\hline \multicolumn{4}{|l|}{ Sex } \\
\hline Female & $182(43.1 \%)$ & $91(43.3 \%)$ & $91(42.9 \%)$ \\
\hline Male & $240(56.9 \%)$ & $119(56.7 \%)$ & $121(57.1 \%)$ \\
\hline \multicolumn{4}{|l|}{ Living } \\
\hline City & $291(69.0 \%)$ & $143(68.1 \%)$ & $148(69.8 \%)$ \\
\hline Rural & $131(31 \%)$ & $67(31.9 \%)$ & $64(30.2 \%)$ \\
\hline \multicolumn{4}{|c|}{ Passive smoking at home } \\
\hline No & $246(58.3 \%)$ & $125(59.5 \%)$ & $121(57.1 \%)$ \\
\hline Father & $76(18.0 \%)$ & $39(18.6 \%)$ & $37(17.5 \%)$ \\
\hline Mother & $32(7.6 \%)$ & $15(7.1 \%)$ & $17(8.0 \%)$ \\
\hline Both & $57(13.5 \%)$ & $26(12.4 \%)$ & $31(14.6 \%)$ \\
\hline Other & $11(2.6 \%)$ & $5(2.4 \%)$ & $6(2.8 \%)$ \\
\hline \multicolumn{4}{|c|}{ School attendance } \\
\hline No & $50(11.8 \%)$ & $24(11.4 \%)$ & $26(12.3 \%)$ \\
\hline Yes & $372(88.2 \%)$ & $186(88.6 \%)$ & $186(87.7 \%)$ \\
\hline \multicolumn{4}{|c|}{ Family atopy } \\
\hline Yes & $320(75.8 \%)$ & $168(80.0 \%)$ & $152(71.7 \%)$ \\
\hline No & $75(17.8 \%)$ & $30(14.3 \%)$ & $45(21.2 \%)$ \\
\hline \multicolumn{4}{|c|}{ Sensitized children } \\
\hline Yes & $210(49.8 \%)$ & $104(49.5 \%)$ & $106(50 \%)$ \\
\hline No & $212(50.2 \%)$ & $106(50.5 \%)$ & $106(50 \%)$ \\
\hline
\end{tabular}

TABLE 2: Number and frequency of children with or without asthma exacerbations during the study.

\begin{tabular}{lccc}
\hline & Placebo & Active treatment & OR $(95 \% \mathrm{IC}) ; p$ \\
\hline No exacerbation & $160(76.2 \%)$ & $193(91.0 \%)$ & $3.17(1.80-5.60) ;<0.001$ \\
At least one exacerbation & $50(23.8 \%)$ & $19(9.0 \%)$ & $207(97.6 \%)$ \\
Less than two exacerbations & $193(91.9 \%)$ & $5(2.4 \%)$ & $3.65(1.32-10.08) ; 0.013$ \\
Two exacerbations & $17(8.1 \%)$ & & \\
\hline
\end{tabular}

TABLE 3: Severity and duration of exacerbations during the study.

\begin{tabular}{lcc}
\hline & Placebo & Active treatment \\
\hline $\begin{array}{l}\text { Total number of exacerbations } \\
\text { Severity }\end{array}$ & 67 & 24 \\
$\quad$ Mild & $21(31.3 \%)$ & $4(16.7 \%)$ \\
$\quad$ Moderate & $44(65.7 \%)$ & $19(79.2 \%)$ \\
$\quad$ Severe & $2(3.0 \%)$ & $1(4.2 \%)$ \\
Duration (days) & $3.3 \pm 2.57$ & $3.3 \pm 2.45$ \\
\hline
\end{tabular}

3.3. Secondary Outcomes. There was a reduction trend for treatment intensity over time (Table 4). No significant difference was observed at the intragroup and intergroup analyses, even if there were some missing data in the database. In particular, more than $40 \%$ of children did not take any treatment at T0. This high figure depended on the asthma severity: more than $80 \%$ of children had intermittent asthma. The frequency of children without treatment was inclined to increase in both groups over time. Table 5 shows the use of oral and inhaled corticosteroids during asthma exacerbations. Oral corticosteroids were prescribed in about $70 \%$ of children, and inhaled corticosteroid dosage was increased in about $50 \%$ of them. So, no significant difference between groups was reported.

3.4. Safety Data. Both treatments were well-tolerated, and no clinically relevant adverse event was reported.

\section{Discussion}

The present study showed that the probiotic mixture containing $B$. breve $\mathrm{B} 632$ and $L$. salivarius LS01 significantly reduced the frequency and severity of asthmatic exacerbations in a primary care setting that accurately represents what occurs in daily practice. 
TABLE 4: Maintenance therapy over time: intensity of asthma treatment in both groups.

\begin{tabular}{ccccccccc}
\hline & \multicolumn{3}{c}{ Placebo } & & \multicolumn{4}{c}{ Active treatment } \\
& No therapy & Low intensity & Mild intensity & High intensity & No therapy & Low intensity & Mild intensity & High intensity \\
\hline T0 & $83(40.5 \%)$ & $40(19.5 \%)$ & $38(18.5 \%)$ & $44(21.5 \%)$ & $95(45.5 \%)$ & $36(17.2 \%)$ & $30(14.4 \%)$ & $48(23.0 \%)$ \\
$T 1$ & $86(42.2 \%)$ & $41(20.1 \%)$ & $37(18.1 \%)$ & $40(19.6 \%)$ & $95(46.6 \%)$ & $33(16.2 \%)$ & $28(13.7 \%)$ & $48(23.5 \%)$ \\
$T 2$ & $99(49.0 \%)$ & $35(17.3 \%)$ & $29(14.4 \%)$ & $39(19.3 \%)$ & $98(48.3 \%)$ & $33(16.3 \%)$ & $31(15.3 \%)$ & $41(20.2 \%)$ \\
$T 3$ & $103(51.5 \%)$ & $33(16.5 \%)$ & $27(13.5 \%)$ & $37(18.5 \%)$ & $104(51.7 \%)$ & $38(18.9 \%)$ & $29(14.4 \%)$ & $30(14.9 \%)$ \\
$T 4$ & $123(61.8 \%)$ & $23(11.6 \%)$ & $25(12.6 \%)$ & $28(14.1 \%)$ & $115(57.2 \%)$ & $32(15.9 \%)$ & $24(11.9 \%)$ & $30(14.9 \%)$ \\
\hline
\end{tabular}

TABLE 5: Treatment during asthma exacerbations in both groups.

\begin{tabular}{lcc}
\hline & Placebo (67 exacerbations) & Active treatment (24 exacerbations) \\
\hline Use of oral corticosteroids & $45(67.2 \%)$ & $17(70.8 \%)$ \\
Increased dosage of inhaled corticosteroids & $34(50.7 \%)$ & $13(54.2 \%)$ \\
\hline
\end{tabular}

Asthma exacerbations represent an important issue in pediatric clinical practice [19]. In particular, asthma exacerbation is closely associated with asthma severity requiring that children require effective management in primary care settings. In childhood, acute upper airway infection, mainly of viral origin, represents the leading cause of asthma relapse [20]. Viral infections implicate bronchial inflammation that triggers airway hyperresponsiveness and further narrows the bronchial lumen. Moreover, allergic subjects are more susceptible to frequent and severe infections than nonallergic subjects [21]. As a result, a vicious circle includes asthma, allergy, infections, and acute respiratory episodes. These phenomena depend on the overexpression of type-2 immune response usually involved in pediatric asthma. It is essential to adopt preventive measures to restore a physiological immune response [22]. The encouraging results here reflect such measures.

A dysbiosis can be generally defined as a reduction in microbial diversity and a combination of the loss of beneficial bacteria such as certain members of the Bacteroides or Firmicutes phyla and a rise in pathobionts (bacteria that become pathogenic under certain conditions), including Proteobacteria. This imbalance may lead to a dysregulated immunological response in several organs, including the lung [23]. Probiotic strains can modulate the immune system by restoring a physiologic type- 1 response, dampening inflammation, and reactivating eubiosis [7]. Examples include a demonstrating the inefficacy of a one-year consumption of fermented milk with Lactobacillus casei in 187 asthmatic preschoolers [24], and another in which Lactobacillus rhamnosus GG use reduced episodes in infants with at least two wheezing episodes and family atopy [25].

Another study showed that eight-week Lactobacillus gasseri A5 supplementation in asthmatic children improved lung function, reduced asthma symptom scores, and increased asthma control test (ACT) score significantly diminishing the production of TNF- $\alpha$, IFN- $\gamma$, IL-12, and IL-13 by peripheral blood mononuclear cells [26]. Some bifidobacteria have also shown benefits, with a combination of
Bifidobacterium longum BB536, Bifidobacterium infantis $\mathrm{M}-63$, and Bifidobacterium breve M-16V, being shown to significantly reduce respiratory symptoms and improve quality of life (QoL), unlike the placebo [27]. One strain, Bifidobacterium lactis Probio-M8, was found to provide added value when ingested along with inhaled Symbicort, by decreasing FeNO, improving ACT, and modulating gut biodiversity [28].

These outcomes are relevant to clinical practice. Asthma exacerbations, mainly if associated with hospitalization, create a burden for the healthcare system and negatively affect children and their families.

The present findings emphasize the importance of selecting strains, since both used here showed positive effects in other disease models [14-17]. The $L$ salivarius strain also can produce tolerogenic peptides, restore the physiological type-1 polarization, expand $\mathrm{T}$ regulatory cells, and improve epithelial barrier function [7-9].

The study does have some limitations. It did not examine longer treatment nor specifically examine mechanistic parameters. In addition, there are many reasons for asthma exacerbation, including respiratory infections, allergen exposure, unauthorized drug withdrawal, exercise, meteorological change, and pollutants. The current study did not analyze the precise reasons for the subjects' asthma exacerbation and the mechanism by which probiotics reduce asthma exacerbations cannot be speculated. Moreover, there were some missing data for some variables, mainly concerning the treatment details. It could happen in studies conducted in a primary care setting.

Nevertheless, the finding that probiotic strains, readily available for consumers, can reduce asthma exacerbations in children provides a potential complementary therapy for primary care physicians.

\section{Conclusions}

The PROPAM study provided clinical evidence suggesting that Bifidobacterium breve B632 (DSM 24706) and 
Ligilactobacillus salivarius LS01 (DSM 22775) may prevent asthma exacerbations in children. Moreover, this probiotic mixture was safe and well-tolerated. At present, the firstline treatment of asthma is still drug-based, but specific strains of probiotics may be auxiliary remedies.

\section{Data Availability}

The clinical data used to support the findings of this study are available from the corresponding author upon request.

\section{Additional Points}

Key Message. Specific strains (Ligilactobacillus salivarius LS01 (DSM 22775) and Bifidobacterium breve B632 (DSM 24706)) may prevent asthma exacerbations in children.

\section{Ethical Approval}

The study was approved by an Ethics Committee. The study was registered: NCT04289441.

\section{Disclosure}

The study's sponsor (Probiotical, Novara, Italy) had no role in study design or data collection. The analyses were carried out before unblinding of data (to be checked on the Authorship basis).

\section{Conflicts of Interest}

MP and AA are employees of Probiotical Research. The other authors have no conflict of interest.

\section{Acknowledgments}

* PROPAM Study Group Members: Francesco Paolo Brunese, Francesco Carlomagno, Donatella Del Gaizo, Antonietta D’Onofrio, Patrizia Gallo, Salvatore Iasevoli, Raffaele Limauro, Roberto Sassi, Giannamaria Vallefuoco. The ADL Farmaceutici (Milan, Italy) provided free samples for the study and supported the study organization.

\section{References}

[1] P. A. B. Wark, J. M. Ramsahai, P. Pathinayake, B. Malik, and N. W. Bartlett, "Respiratory viruses and asthma," Seminars in Respiratory and Critical Care Medicine, vol. 39, no. 1, pp. 45-55, 2018.

[2] M. C. Maciag and W. Phipatanakul, "Prevention of asthma: targets for intervention," Chest, vol. 158, no. 3, pp. 913-922, 2020.

[3] M. Aw, J. Penn, G. M. Gauvreau, H. Lima, and R. Sehmi, "Atopic march: collegium Internationale Allergologicum Update 2020," International Archives of Allergy and Immunology, vol. 181, no. 1, pp. 1-10, 2020.

[4] T. Haahtela, "A biodiversity hypothesis," Allergy, vol. 74, pp. 1445-1456, 2019.

[5] K. Hufnagl, I. Pali-Schöll, F. Roth-Walter, and E. Jensen-Jarolim, "Dysbiosis of the gut and lung microbiome has a role in asthma," Seminars in Immunopathology, vol. 42, no. 1, pp. 75-93, 2020.

[6] A. T. Dang and B. J. Marsland, "Microbes, metabolites, and the gut-lung axis," Mucosal Immunology, vol. 12, no. 4, pp. 843850, 2019.

[7] I. Balta, E. Butucel, V. Mohylyuk et al., "Novel insights into the role of probiotics in respiratory infections, allergies, cancer, and neurological abnormalities," Diseases, vol. 9, no. 3, p. 60, 2021.

[8] C. Maldonado Galdeano, S. I. Cazorla, J. M. Lemme Dumit, E. Velez, and G. Perdigon, "Beneficial effects of probiotic consumption on the immune system," Annals of Nutrition and Metabolism, vol. 74, no. 2, pp. 115-124, 2019.

[9] Z. Al Nabhani and G. Eberl, "Imprinting of the immune system by the microbiota early in life," Mucosal Immunology, vol. 13, no. 2, pp. 183-189, 2020.

[10] J. Lin, Y. Zhang, C. He, and J. Dai, “Probiotics supplementation in children with asthma: a systematic review and metaanalysis," Journal of Paediatrics and Child Health, vol. 54, no. 9, pp. 953-961, 2018.

[11] X. Du, L. Wang, S. Wu et al., "Efficacy of probiotic supplementary therapy for asthma, allergic rhinitis, and wheeze: a metaanalysis of randomized controlled trials," Allergy and Asthma Proceedings, vol. 40, pp. 250-260, 2019.

[12] X. Wei, P. Jiang, J. Liu, R. Sun, and L. Zhu, "Association between probiotic supplementation and asthma incidence in infants: a meta-analysis of randomized controlled trials," The Journal of Asthma, vol. 57, no. 2, pp. 167-178, 2020.

[13] L. Meirlaen, E. I. Levy, and Y. Vandenplas, "Prevention and management with pro-, pre- and synbiotics in children with asthma and allergic rhinitis: a narrative review," Nutrients, vol. 13, p. 934, 2021.

[14] E. Iemoli, D. Trabattoni, S. Parisotto et al., "Probiotics reduce gut microbial translocation and improve adult atopic dermatitis," Journal of Clinical Gastroenterology, vol. 46, pp. S33-S40, 2012.

[15] E. Nettis, E. Di Leo, A. Pastore et al., "Probiotics and refractory chronic spontaneous urticaria," European Annals of Allergy and Clinical Immunology, vol. 48, pp. 182-187, 2016.

[16] M. C. Fortuna, V. Garelli, G. Pranteda et al., "A case of scalp rosacea treated with low dose doxycycline and probiotic therapy and literature review on therapeutic options," Dermatologic Therapy, vol. 29, no. 4, pp. 249-251, 2016.

[17] L. Drago, E. de Vecchi, A. Gabrieli, R. de Grandi, and M. Toscano, "Immunomodulatory effects of Lactobacillus salivarius LS01 and Bifidobacterium breve BR03, alone and in combination, on peripheral blood mononuclear cells of allergic asthmatics," Immunologic Research, vol. 7, no. 4, pp. 409$413,2015$.

[18] L. Indinnimeo, M. M. del Giudice, E. Chiappini et al., "Gestione dell'attacco acuto di asma in età pediatrica," Area Pediatrica, vol. 18, no. 2, pp. 4-9, 2017.

[19] H. E. Hoch, P. R. Houin, and P. C. Stillwell, "Asthma in children: a brief review for primary care providers," Pediatric Annals, vol. 48, no. 3, pp. e103-e109, 2019.

[20] M. Haktanir Abul and W. Phipatanakul, "Severe asthma in children: evaluation and management," Allergology International, vol. 68, no. 2, pp. 150-157, 2019.

[21] G. Ciprandi, M. A. Tosca, and L. Fasce, "Allergic children have more numerous and severe respiratory infections than nonallergic children," Pediatric Allergy and Immunology, vol. 17, no. 5, pp. 389-391, 2006. 
[22] L. Indinnimeo, D. Porta, F. Forastiere et al., "Prevalence and risk factors for atopic disease in a population of preschool children in Rome: challenges to early intervention," International Journal of Immunopathology and Pharmacology, vol. 29, pp. 308-319, 2016.

[23] W. Barcik, R. C. T. Boutin, M. Sokolowska, and B. B. Finlay, "The role of lung and gut microbiota in the pathology of asthma," Immunity, vol. 52, no. 2, pp. 241-255, 2020.

[24] M. Giovannini, C. Agostoni, E. Riva et al., "Clinical investigation-a randomized prospective double blind controlled trial on effects of long-term consumption of fermented milk containing Lactobacillus casei in pre-school children with," Pediatric Research, vol. 62, no. 2, pp. 215-220, 2007.

[25] M. A. Rose, F. Stieglitz, A. Köksal, J. Schubert, J. Schulze, and S. Zielen, "Efficacy of probiotic lactobacillus GG on allergic sensitization and asthma in infants at risk," Clinical and Experimental Allergy, vol. 40, no. 9, pp. 1398-1405, 2010.

[26] Y.-S. Chen, Y. L. Lin, R. L. Jan, H.-H. Chen, and J.-Y. Wang, "Randomized placebo-controlled trial of lactobacillus on asthmatic children with allergic rhinitis," Pediatric Pulmonology, vol. 45, no. 11, pp. 1111-1120, 2010.

[27] M. Miraglia del Giudice, C. Indolfi, M. Capasso, N. Maiello, F. Decimo, and G. Ciprandi, "Bifidobacterium mixture (B. longum BB536, B. infantis M-63, B. breve M-16V) treatment in children with seasonal allergic rhinitis and intermittent asthma," Italian Journal of Pediatrics, vol. 43, no. 1, p. 25, 2017.

[28] A. Liu, T. Ma, N. Xu et al., "Adjunctive probiotics alleviates asthmatic symptoms via modulating the gut microbiome and serum metabolome," Microbiology Spectrum, vol. 9, no. 2, article e00859-21, 2021. 